\title{
GTIKF- Gabor-Transform Incorporated K-Means and Fuzzy C Means Clustering for Edge Detection in CT and MRI
}

\author{
Dr. Akey Sungheetha \\ Data Science SIG member, \\ Computer Science and Engineering, School of Electrical Engineering and Computing, \\ Adama Science and Technology University, Adama, Nazret, Ethiopia \\ Dr. Rajesh Sharma R \\ Image Processing SIG member, \\ Computer Science and Engineering, School of Electrical Engineering and Computing, \\ Adama Science and Technology University, Adama, Nazret, Ethiopia
}

\begin{abstract}
The detection of edges is the one of the important stage in the application, associated with the machine vision, computer vision and the image processing. It is most commonly and highly preferred in the area were the extraction or the detection of the attribute are necessary. As the manual methods of diagnosis in the medical images acquired from the CT (computed tomography) and the MRI (magnetic resonance images) are very tedious and as well as time consuming, the paper puts forth the methodology to detect the edges in the CT and the MRI by employing Gabor Transform as well as the soft and the hard clustering. This proposed method is highly preferred among the image with dynamic variations. The technique used in the paper is evaluated using 4500 instance of the MRI and 3000 instance of CT. The results on the basis of the figure of merit (FOM) and Misclassification rate (MCR) are compared with other standard approaches and the performance was evinced.
\end{abstract}

Keywords: Hard and Soft clustering, Edge detection, Image Processing, CT and MRI

\section{Introduction}

The process of detecting edges is a carefully worked-out procedure, and primary tools in the handing out of images to detect the edges in the images were there are dynamic variations in its luminosity or the discontinuities in the picture. The edge detection process is most commonly preferred in the area were the extraction of the images or the detection is requisite. It is described as one of the stages in the in the wide range of applications related to the computer vision, image processing and machine vision.

The images acquired from the CT and the MRI are predominantly used process to deliver the images of the disease affected parts as well as the anatomical structures. The purpose of the tissue properties takes a 
Journal of Soft Computing Paradigm (JSCP) (2020)

Vol.02/ No. 02

Pages: 111-119

http://irojournals.com/jscp/

DOI: https://doi.org/10.36548/jscp.2020.2.004

significant part in the diagnosis through the therapeutic pictures to recognize the "difference in the anatomical structures and the tissues, this enables the physician to identify the affected areas without any difficulties." But as this process is manual process is tedious and time consuming.

To minimize the weariness caused by the manual operations. The edge detection process was introduced. Many conventional edge detection methodologies such as the "sobel gradient detector" [1] and [2] used for the purpose designing an edge detection filter "with the chip being designed based on a 2-mu m, double metal, CMOS technology and was implemented using the silicon compiler system in less than 2 manmonths. It is designed to operate with a $10 \mathrm{MHz}$." and "utilizes the sobel technique to identify the edges using the edge detection algorithm it returns the edges at those points where the gradient of the considered image is maximum." "The laplacian operator which is a second derivative operator is often used in the detection of edges as they were performed well and yielded better results than the edge detectors based on the first derivative. But the laplacians were very sensitive to the noise" [3]. The over view of the various edge detectors along with the advantages and disadvantages was presented in [4].

So to eradicate the problems in the so for found in the edge detection the Gabor wavelet transform was introduced. The applications of the Gabor wavelet transform was discussed in [5]. Jian, et al [8] in his paper has devised Zhitao, [7] in his paper presented the research on the Gabor Wavelet and its uses in the image detection. The Gabor transform has a filter that operates based on the "frequency and the orientation representations". The filter subdues the noises and identifies the edges. The proposed method utilizes the Gabor filter to detect the edges. The clustering technique is utilized for transforming the images in the gray scale to binary. The noise information's in the images are removed and the edges are preserved using the median or the bilateral filter. This minimizes the noise at a very less time and are more compatible for the real life applications. The basic processing on the images is segregated into following steps. They are

i. Segmentation

ii. Identifying the edge points

iii. De-noising /Thresholding (adaptive median filter/ adaptive threshold value)

iv. Application of filter bank (application of Gabor Wavelet Transform or any other classical method)

The "Gabor Wavelet Transform" as well as the clustering methods are used in the paper to over the prevailing issues in the edge detection and improve its performance. The basics of edge detection and the parameters involved in the assessment of the edge detection is as follows

ISSN: 2582-2640 (online) 
Journal of Soft Computing Paradigm (JSCP) (2020)

Vol.02/ No. 02

Pages: 111-119

http://irojournals.com/jscp/

DOI: https://doi.org/10.36548/jscp.2020.2.004

a) Misclassification Rate (MCR)

b) Figure of Merit

These parameter enable one to measure the identity or the equivalence among the edge detection that is made manually and by using the algorithm. The MCR is estimated according to the equation 1

$$
M C R=\frac{\sum \bmod \left(B G_{a c} \cap B G_{d e l}\right)+\sum \bmod \left(F G_{a c} \cap F G_{d e l}\right)}{\sum\left(B G_{a c} \cap F G_{d e l}\right)} * 100 \%
$$

$F G$ is the fore ground, $B G$ is the back ground, $a c$ is the actual and $d e l$ is the detected images, the detection rate and the MCR value are inversely proportional, the detection \% increases as the MCR value decreases. The figure of merit is calculated according to the equation 2

$$
F O M=\frac{1}{\max \left(E_{d}, E_{a c c}\right)} \sum_{x=1}^{E_{d}} \frac{1}{1+a \text { dist }\left(E_{a c c i}, E_{d}\right)^{\wedge} 2}
$$

Where the $E_{d}, E_{a c c}$ are the amount of the detected and the accurate edges and $a$ is the scaling factor that is equal to $\frac{1}{9}$ and $\operatorname{dist}\left(E_{a c c i}, E_{d}\right)$ is the distance between the accurate edges and the detected, if edge detected than the FOM denotes 1 other wise denotes zero. The figure. 1 below shows the process involved in the proposed method for detecting edges.

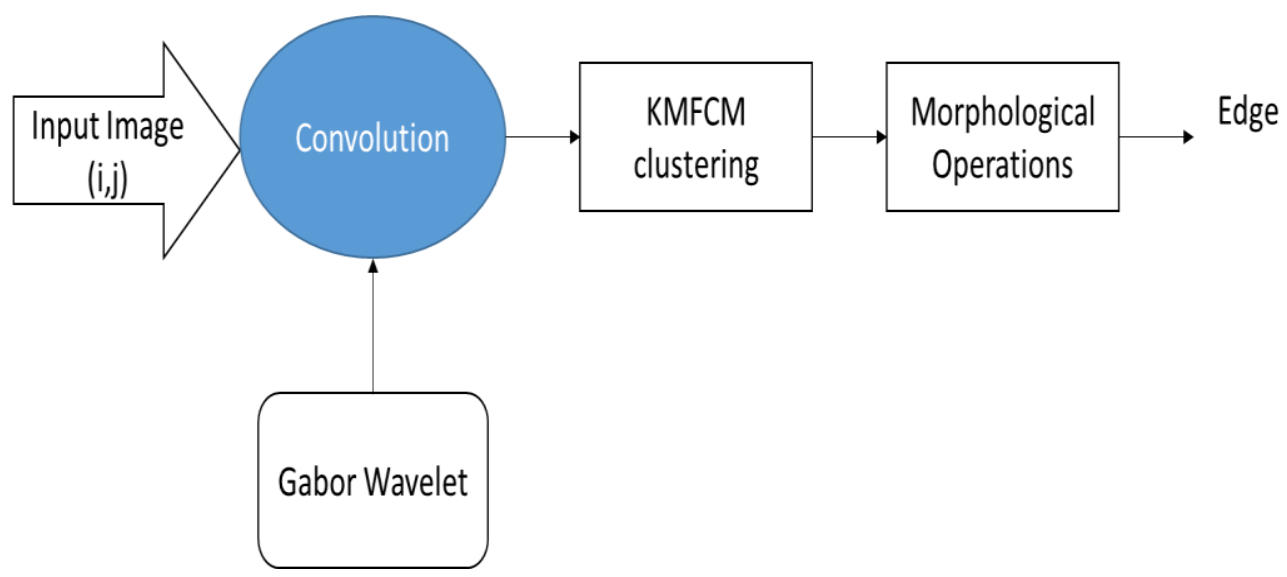

Figure.1 Edge Detection Procedure Proposed 
Journal of Soft Computing Paradigm (JSCP) (2020)

Vol.02/ No. 02

Pages: 111-119

http://irojournals.com/jscp/

DOI: https://doi.org/10.36548/jscp.2020.2.004

The proposed method of edge detection is laid out with 2.Related Works, 3. Proposed Work, 4.Results evaluation and 5. Conclusion.

\section{Related works}

Raj, J. S. et al [8] used the process of fuzzy and the means in the identify the routes for information conveyance in the wireless sensor networks, Joseph, S. I. T. et al [9] elaborated the data mining techniques available to have an intelligent computing system. Ananthi, J. V. et al [10] put forth a "Recurrent Neural Networks and Nonlinear Prediction in Support Vector Machines" to enhance the detection capabilities.

Manoharan, S. et al [11] conducted a study on the feature detection using the "Hermitian Graph Wavelets" and Sathesh, A. et al [12] put forth the "Performance Analysis of Granular Computing Model in Soft Computing Paradigm for Monitoring of Fetal Echocardiography"

Bezdek, James C et al [13]., presented the tutorial on the "fuzzy c means clustering" in the year 1984 and Alsabti, et al [14] presented an "An efficient k-means clustering algorithm" in the year 1997, the proposed method involves the Gabor wavelet and the soft (the fuzzy c means) and hard the (k-means) clustering to enhance the edge detection capabilities in the MSI and CT.

\section{Proposed Algorithm}

The planned procedure uses the Gabor wavelet Transform to remove the noise information and conserve the edges. The K-means is used to separate the identical features into the small number of groups. To represent the features of the images in the form of histogram properties the proposed method utilizes the Fuzzy c means clustering of images. The figure .2 below shows the flow diagram of the proposed method. 
Journal of Soft Computing Paradigm (JSCP) (2020)

Vol.02/ No. 02

Pages: 111-119

http://irojournals.com/jscp/

DOI: https://doi.org/10.36548/jscp.2020.2.004

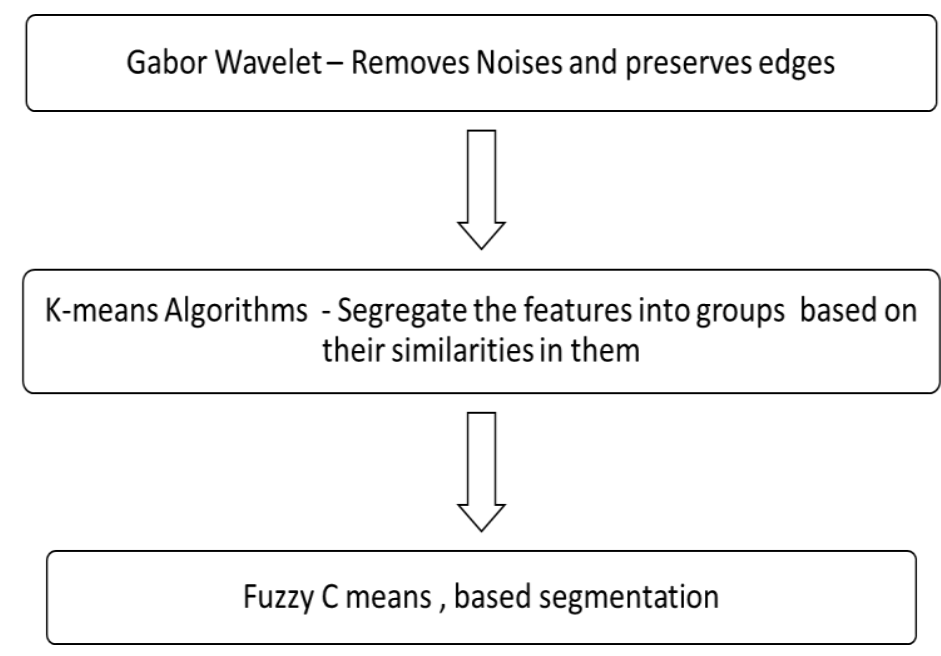

Figure .2 Proposed Flow Diagram

The planned scheme as exposed in the figure.1 utilizes Gabor filters (linear filter) for detecting the edges of the images. This filter is similar to the vision system of the humans and are capable of delivering the rate of recurrence and alignment illustrations of the Gabor filters specifically in the examination and discernment of texture. This is also depicted as the function of the "Gaussian kernel alleviator" by the sinusoidal wave. It regulates the frequency attributes to remove the noises in the MRI or the CT and conserves the information's about the edges. As shown in the figure.1 the harmonics of the FT (Fourier transform) and the GFT Gaussian-Fourier transform are convoluted using the "multiplication- convolution property" and delivers the FT of Gabor filter. "The filter has orthogonal directions in terms of real and imaginary components" the discrimination of the two dimensional Gabor wavelets are accurate to get the features related to the directions and the wave forms in order to conserve the edge information's minimizing the disturbances in the images. The input image fed in the two dimensional form and convoluted and reaches the output in the form "sinusoidal wave frequency", "sinusoidal wave orientation" and standard deviation.

Now the similar features are clustered to gather using the k-means algorithms, the algorithm scopes to allot the set of identical information to the cluster the minimum depth across the cluster and the features are computed using the Euclidean distance. The observations ( $\mathrm{X} 1$ to $\mathrm{Xn}$ ) is segregated across the ' $\mathrm{K}$ ' clusters to minimize the "sum of squared depth within the clusters" according to equation 3

Mean square error of the Cluster

= summation of squared difference across the observation and the clusters 
Journal of Soft Computing Paradigm (JSCP) (2020)

Vol.02/ No. 02

Pages: 111-119

http://irojournals.com/jscp/

DOI: https://doi.org/10.36548/jscp.2020.2.004

Mean square error is evaluated to allocate the clusters. Whenever the difference across the cluster and the observation less the observation is allotted to the cluster. For this the mid position is initialized and the observation are allotted to the closest cluster by this the every pixels in the image becomes a member to its closest cluster.

Further to segment the images the fuzzy c means clustering is used. This is basically done to represent the image features in its histogram attributes. The segmentation using the FCM algorithm conserves maximum information than any other standard approaches. This is almost similar to the K-means, but every features vectors are valued by using the member ship function [0 to 1] as the algorithm totally relies on the criteria of the depth count across the clusters/ on the "point prototype clustering model " and attains a partition optimized center point. This transforms the images in the gray scale to binary. The unassociated parts or information's in the image are morphed using the morphological operations.

\section{Results Evaluation}

The process is evaluated using various instance of CT and the MRI, the procedure is validated with 4500 and 3000 instance of the magnetic resonance images and CT respectively of different parts of the human body. The edge detection for the brain, heart and the breast images through MRI and the chest belly and the pelvis images through CT is applied using the Gabor followed by the clustering techniques hard and soft.

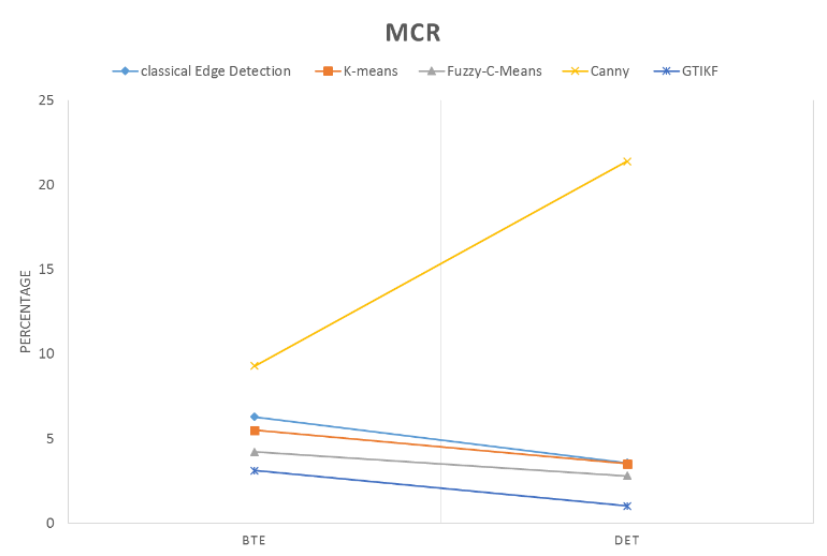

Figure.3 Misclassification Rate

ISSN: 2582-2640 (online) 
Journal of Soft Computing Paradigm (JSCP) (2020)

Vol.02/ No. 02

Pages: 111-119

http://irojournals.com/jscp/

DOI: https://doi.org/10.36548/jscp.2020.2.004

The figure. 3 is the misclassification results comparison for the procedure laid out in the paper and other standard methods. Such as individual K-means, Fuzzy c means, Classical edge detection methods canny edge detection etc. The results are observed for both the basic true edges (BTE) and the detected images (DET)

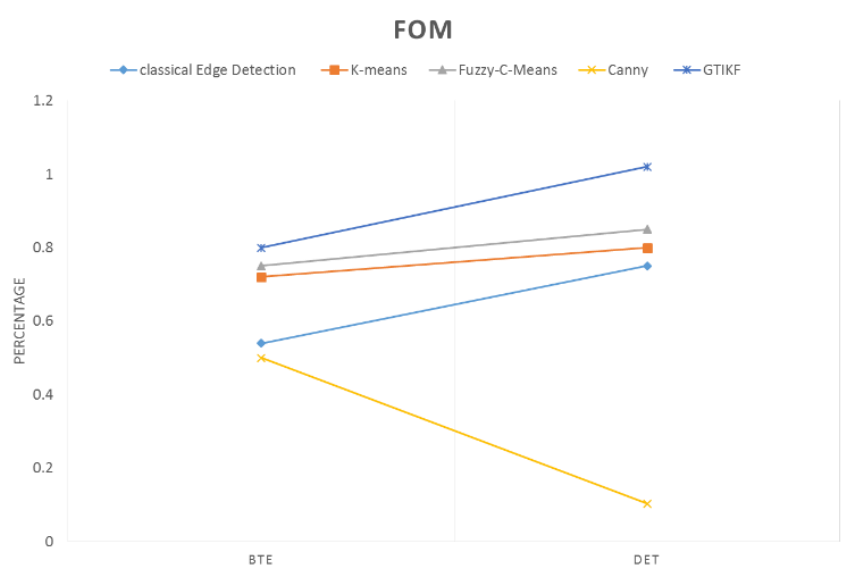

Figure.4 Figure of Merit

The figure. 4 is the figure of merit and the comparison observed $\mathrm{f}$ for the procedure laid out in the paper and other standard methods. Such as individual K-means, Fuzzy c means, Classical edge detection methods canny edge detection etc. The results are observed for both the basic true edges (BTE) and the detected images (DET)

\begin{tabular}{|c|c|c|c|c|c|c|c|c|c|c|c|}
\hline \multirow[t]{2}{*}{ Type of Edges } & \multirow[t]{2}{*}{ PSNR } & \multicolumn{5}{|l|}{ MCR } & \multicolumn{5}{|l|}{ FOM } \\
\hline & & Kmeans & Classical & $\mathrm{FCM}$ & Canny & GTKFF & Kmeans & Classical & $\mathrm{FCM}$ & Canny & GTIKF \\
\hline Basic & 30.56 & 5.57 & 7.8 & 5,7 & 9.67 & 2 & .76 & .45 & .58 & .12 & 1.09 \\
\hline Detected & 27.89 & .98 & 1,54 & .95 & 17.45 & .45 & .56 & .32 & .59 & .04 & 1.67 \\
\hline
\end{tabular}

Table.1 Comparison of Results 
Journal of Soft Computing Paradigm (JSCP) (2020)

Vol.02/ No. 02

Pages: 111-119

http://irojournals.com/jscp/

DOI: https://doi.org/10.36548/jscp.2020.2.004

The table .1 provides the overview of the different results observed for the different algorithm for the basic as well as the detected images along with the PSNR value. The results prove that the GTIKF is much better compared to the other standard approaches.

\section{Conclusion}

The enhance the process of detecting edges even in the noisy environment the proposed method has utilized the GTIKF, Gabor transform integrated with the K means and the Fuzzy c means. The proposed method was validated utilizing the instance of MRI as well as CT, the results observed were convincing and better compared to the other state of approaches. But the method also has a draw back the Fuzzy c means are very slow compared to the $\mathrm{K}$ means and are sensitive to noise if the spatial information's alone are considered. To overcome this the proposed method in the future the paper is to proceed using the bio inspired or the evolutionary based algorithms to be used in the process of edge detection.

\section{References}

[1] Kanopoulos, Nick, Nagesh Vasanthavada, and Robert L. Baker. "Design of an image edge detection filter using the Sobel operator." IEEE Journal of solid-state circuits 23, no. 2 (1988): 358-367.

[2] Gupta, Samta, and Susmita Ghosh Mazumdar. "Sobel edge detection algorithm." International journal of computer science and management Research 2, no. 2 (2013): 1578-1583.

[3] Wang, Xin. "Laplacian operator-based edge detectors." IEEE transactions on pattern analysis and machine intelligence 29, no. 5 (2007): 886-890.

[4] Shrivakshan, G. T., and Chandramouli Chandrasekar. "A comparison of various edge detection techniques used in image processing." International Journal of Computer Science Issues (IJCSI) 9, no. 5 (2012): 269.

[5] Manjunath, B. S. "Gabor wavelet transform and application to problems in early vision." In ASILOMAR CONFERENCE ON SIGNALS SYSTEMS AND COMPUTERS, pp. 796-796. COMPUTER SOCIETY PRESS, 1992.

[6] Jian, Muwei, and Lei Liu. "Texture image classification using visual perceptual texture features and gabor wavelet." Journal of computers 4, no. 8 (2009): 763.

[7] Zhitao, Xiao, Guo Chengming, Yu Ming, and Li Qiang. "Research on log Gabor wavelet and its application in image edge detection." In 6th International Conference on Signal Processing, 2002., vol. 1, pp. 592-595. IEEE, 2002.

[8] Raj, J. S. (2019). QOS Optimization of Energy Efficient Routing In Iot Wireless Sensor Networks. Journal of ISMAC, 1(01), 12-23. 
Journal of Soft Computing Paradigm (JSCP) (2020)

Vol.02/ No. 02

Pages: 111-119

http://irojournals.com/jscp/

DOI: https://doi.org/10.36548/jscp.2020.2.004

[9] Joseph, S. I. T. (2019). Survey of Data Mining Algorithm's For Intelligent Computing System. Journal of trends in Computer Science and Smart technology (TCSST), 1(01), 14-24.

[10] Raj, J. S., \& Ananthi, J. V. (2019). Recurrent Neural Networks and Nonlinear Prediction in Support Vector Machines. Journal of Soft Computing Paradigm (JSCP), 1(01), 33-40.

[11] Manoharan, S. (2019). Study On Hermitian Graph Wavelets in Feature Detection. Journal of Soft Computing Paradigm (JSCP), 1(01), 24-32.

[12] Sathesh, A. (2019). Performance Analysis of Granular Computing Model in Soft Computing Paradigm For Monitoring Of Fetal Echocardiography. Journal of Soft Computing Paradigm (JSCP), 1(01), 14-23

[13] Bezdek, James C., Robert Ehrlich, and William Full. "FCM: The fuzzy c-means clustering algorithm." Computers \& Geosciences 10, no. 2-3 (1984): 191-203.

[14] Alsabti, Khaled, Sanjay Ranka, and Vineet Singh. "An efficient k-means clustering algorithm." (1997).

\section{Author's Biography}

Dr. Akey Sungheetha is a Data Science SIG member, in the department of Computer Science and Engineering, in School of Electrical Engineering and Computing, in Adama Science and Technology University, Adama, Nazret, Ethiopia her major area of research includes Computer Networks, Chaos Theory, Particle Swarm Optimization, Probabilistic Computing, Fuzzy, Bio- Inspired Computing, Data Visualization, Fault Diagnosis.

Dr. Rajesh Sharma $\mathrm{R}$ is an Image Processing SIG member, in Computer Science and Engineering department, School of Electrical Engineering and Computing, Adama Science and Technology University, Adama, Nazret, Ethiopia her major area of research includes Probabilistic Computing, Fuzzy, Bio- Inspired Computing, Data Visualization, Fault Diagnosis, Robotics, Internet of Things, Neuro Computing, Information Retrieval Human-Machine Interface and Network Security 